\title{
POSITIVE INCREASING SOLUTIONS OF QUASILINEAR DYNAMIC EQUATIONS
}

\author{
E. AKIN-BOHNER
}

Abstract. We consider quasilinear dynamic equations whose solutions are classified into disjoint subsets by certain integral conditions. In particular, we investigate the asymptotic behavior of all positive increasing solutions of quasilinear dynamic equations.

Mathematics subject classification (2000): 39A10.

Key words and phrases: measure chains, time scales, quasilinear equations, half-linear equations, Sturm-Liouville equations.

\section{REFERENCES}

[1] E. AKIN, Boundary value problems for a differential equation on a measure chain, Panamer. Math. J., 10, (2000), 17-30.

[2] E. AKIN-Bohner, Positive decreasing solutions of quasilinear dynamic equations, Math. Comput. Modelling, 43, (2006), 283-293.

[3] M. BohneR, G. GuSEINOV, Improper integrals on time scales, Dyn. Syst. Appl., 12, (2003), 45-65.

[4] M. Bohner, A. Peterson, Dynamic Equations on Time Scales: An Introduction with Applications, Birkhäuser, Boston, 2001.

[5] M. Bohner, A. Peterson, Advances in Dynamic Equations on Time Scales, Birkhäuser, Boston, 2003.

[6] M. Bohner, C. Tisdell, Second order dynamic inclusions, J. Nonlinear Math. Phys, 12, (2) (2005), $36-45$.

[7] M. CECCHI, Z. DOŠLÁ AND M. MARINI, Unbounded solutions of quasilinear difference equations, Comp. Math. Appl., 45, (6-9) (2003), 1113-1123.

[8] M. CECCHI, Z. DoŠLÁ AND M. MARINI, On nonoscillatory solutions of differential equations with p-Laplacian, Advances of Math. Sci. and Appl., 11, (1) (2001), 419-436.

[9] M. CECCHI, Z DoŠLÁ AND M. MARINI, Positive decreasing solutions of quasilinear difference equations, Comp. Math. Appl., 42, (2001), 1401-1410.

[10] J. I. DiAZ, Nonlinear partial differential equations and free boundaries, vol. 1: Elliptic equations. Pitman Advanced Publ., 106, 1985.

[11] M. R. Grossinho, P. OMARI, A multiplicity result for a class of quasilinear elliptic and parabolic problems, Electron. J. Diff. Eqns., 8, (1997), 1-16.

[12] I. T. Kiguradze, T. A. Chanturia, Asymptotic Properties of Solutions of Nonautonomous Ordinary Differential Equations, Kluwer Academic Publishers, Netherlands, 1993. 